\section{Pobreza y desigualdades en el sector de la salud ${ }^{1,2}$}

\author{
Adam Wagstaff ${ }^{3}$
}

\section{RESUMEN}

La pobreza y la mala salud son fenómenos interrelacionados. Los países pobres tienden a presentar peores resultados sanitarios que los más pudientes, y dentro de cada país las personas pobres tienen más problemas de salud que las acomodadas. Esta asociación refleja una relación de causalidad que funciona en los dos sentidos: la pobreza genera mala salud, y la mala salud hace que los pobres sigan siendo pobres. En este artículo se examinan las pruebas disponibles respecto a las desigualdades sanitarias entre las personas pobres y las que no lo son, así como respecto a las repercusiones que en forma de empobrecimiento y desigualdad de ingresos pueden tener los gastos en atención de salud. Se hace una exposición sucinta de los actuales conocimientos sobre las causas de las desigualdades y sobre la eficacia de las políticas destinadas a combatirlas, y se señala que es demasiado poco lo que se sabe acerca de los efectos de esas políticas, pese a las abundantes técnicas de medición disponibles y a los muchos datos obtenidos sobre la magnitud y las causas de las desigualdades.

Palabras clave: pobreza, estado de salud, ingresos, accesibilidad a los servicios de salud, organización del financiamiento, justicia social.

\footnotetext{
Basado en: Wagstaff A. Poverty and health. (CMH Working Paper Series, Paper No. WG1: 5). Disponible en: http://www.cmhealth. org/wg1_paper5.pdf. Los resultados, interpretaciones y conclusiones son exclusivamente del autor, y no representan necesariamente los puntos de vista del Banco Mundial, de sus directores ejecutivos o de los países que representan.

2 Publicado originalmente en inglés (Bull World Health Organ 2002; 80:97-105) y traducido al español con el permiso del propietario del copyright: Organización Mundial de la Salud.

3 Banco Mundial, Washington, D.C. La correspondencia debe enviarse al autor a la siguiente dirección: The World Bank, 1818 H Street NW, Washington, D.C. 20433, Estados Unidos de América. Correo electrónico: awagstaff@worldbank.org
}

La pobreza y la mala salud están interrelacionadas. Los países pobres tienden a presentar peores resultados sanitarios que los más pudientes y, dentro de cada país, las personas pobres tienen más problemas de salud que las acomodadas. La asociación entre la pobreza y la mala salud refleja una relación de causalidad bidireccional. La enfermedad o la fecundidad excesivamente alta pueden tener un considerable efecto en los ingresos familiares $(1,2)$ y marcar incluso la diferencia entre estar por encima o por debajo de la línea de pobreza (3). Además, la mala salud se asocia frecuentemente a considerables costos de atención sanitaria (4). Pero la pobreza y los bajos ingresos también son causa de mala salud (5). Los países pobres y las personas pobres sufren múltiples privaciones que se expresan en altos niveles de mala salud $(6,7)$. De este modo, las personas pobres se ven atrapadas en un círculo vicioso: la pobreza engendra mala salud y la mala salud mantiene la pobreza (figura 1).

Varias de las principales organizaciones internacionales y donantes bilaterales tienen ahora como objetivo primario mejorar los resultados de salud de los pobres del mundo (8-10). Esto refleja una tendencia creciente de esas organizaciones a definir sus objetivos en términos de reducción de la pobreza $(11,12)$ y la interpretación cada vez más amplia que se está haciendo del término "pobreza" $(6,13)$. Sin embargo, también refleja un consenso creciente de que las desigualdades entre los ricos y los pobres con respecto a los resultados sanitarios son injustas (14), no porque los pobres sean de algún modo más merecedores que los que tienen más dinero, sino porque estas desigualdades corresponden obviamente a las limitaciones y oportunidades muy diferentes que tienen unos y otros, y no a una tendencia de los dos grupos a hacer elecciones diferentes (15-20). Los efectos perjudiciales de la mala salud sobre el nivel de vida familiar también están siendo considerados cada vez más como una cuestión de justicia social, posiblemente como reflejo del punto de vista de que las pérdidas de ingresos y los pagos de la atención sanitaria asociados a la mala salud son simplemente la consecuencia, involuntaria, de "choques" sanitarios no deseados (21). Esto distingue a los gastos sanitarios de la mayor parte de las demás partidas de los presupuestos familiares y conduce naturalmente a la idea de que la carga financiera de dichos choques debería ser soportada por la comunidad en su conjunto, en vez de permitir que tenga 
FIGURA 1. Ciclo de la salud y la pobreza

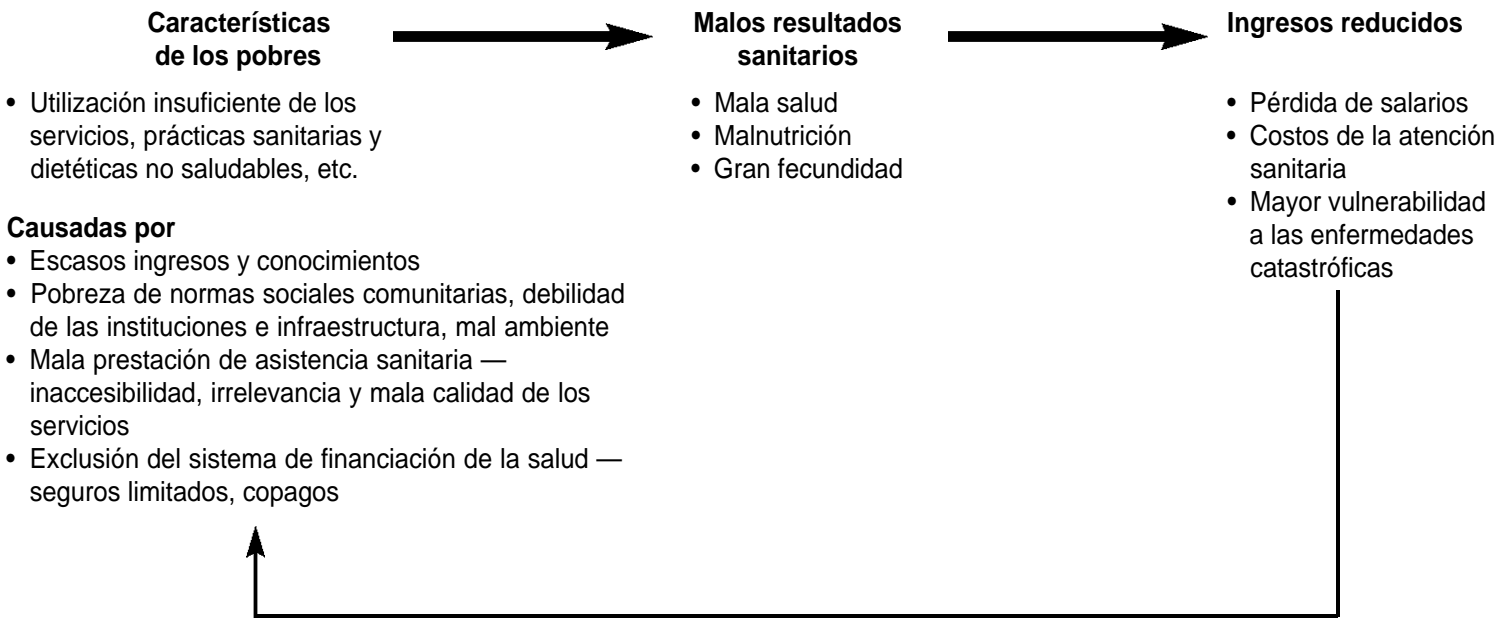

una repercusión negativa sobre la desigualdad de ingresos y la pobreza. En varios países de la Organización para la Cooperación y el Desarrollo Económico (OCDE) (22), y otros (23), parece haber una aceptación de la idea de que tanto los pagos de bolsillo como los pagos destinados a los planes de protección deberían estar relacionados con los ingresos familiares, idea que ha sido defendida recientemente por la Organización Mundial de la Salud (24).

Este trabajo proporciona una perspectiva general de las investigaciones relacionadas con las desigualdades en el campo de la salud que colocan a los pobres en desventaja y con los cambios en el empobrecimiento y la desigualdad de ingresos generados por el pago de la atención sanitaria. No se tratará el tema más amplio del empobrecimiento asociado a la pérdida de ingresos por mala salud, porque la creación de planes de protección frente a tales pérdidas va más allá del campo de la política sanitaria tal como se entiende hoy día. No obstante, cabe señalar que los ingresos perdidos probablemente sean una causa de empobrecimiento más importante que los pagos de bolsillo por los servicios de salud (25). Se analizan los datos relacionados con las desigualdades sanitarias y el empobrecimiento, así como los factores que rigen los resultados y la eficacia de las políticas en estos campos.

\section{DATOS SOBRE LAS DESIGUALDADES SANITARIAS ENTRE LOS POBRES Y LOS NO POBRES}

En Europa ha habido una larga tradición de medición de las desigualdades socioeconómicas en el campo de la salud que abarca tanto la metodolo- gía (26-28) como los análisis empíricos (29-37). En otras regiones del mundo, y especialmente en el mundo en desarrollo, se han efectuado menos trabajos empíricos sobre el tema (38-42).

Vale la pena destacar los siguientes hallazgos clave de la literatura sobre los datos empíricos. Primero, las desigualdades sanitarias son casi siempre en perjuicio de los pobres. Los pobres tienden a morir antes y a presentar mayores niveles de morbilidad que quienes tienen más dinero.

Segundo, las desigualdades tienden a ser más pronunciadas con los indicadores objetivos de mala salud, como las medidas antropométricas de malnutrición y la mortalidad, que con los indicadores subjetivos. A menudo se observa que en los países en desarrollo estos últimos producen a veces gradientes perversos, de modo que los pudientes dicen tener peor salud que los pobres (43). Pero esto tiende a ocurrir con los indicadores que dependen mucho de la influencia de factores transitorios, como si el encuestado ha estado enfermo en las dos semanas anteriores. En los países industrializados se observa un comportamiento similar de estos indicadores (44). Tanto en el mundo en desarrollo (45) como en el mundo industrializado $(31,36,46)$, los indicadores de enfermedad a más largo plazo, como las enfermedades de larga duración, la limitación de actividades importantes o la autoevaluación de la salud, tienden a presentar desigualdades que son desventajosas para los pobres.

Tercero, hay grandes variaciones entre los países con respecto a la magnitud de las desigualdades sanitarias, aunque estas variaciones en sí mismas también varían según los indicadores sanitarios y socioeconómicos utilizados. Por ejemplo, en América Latina las desigualdades entre los po- 
FIGURA 2. Marco conceptual para entender las desigualdades en salud

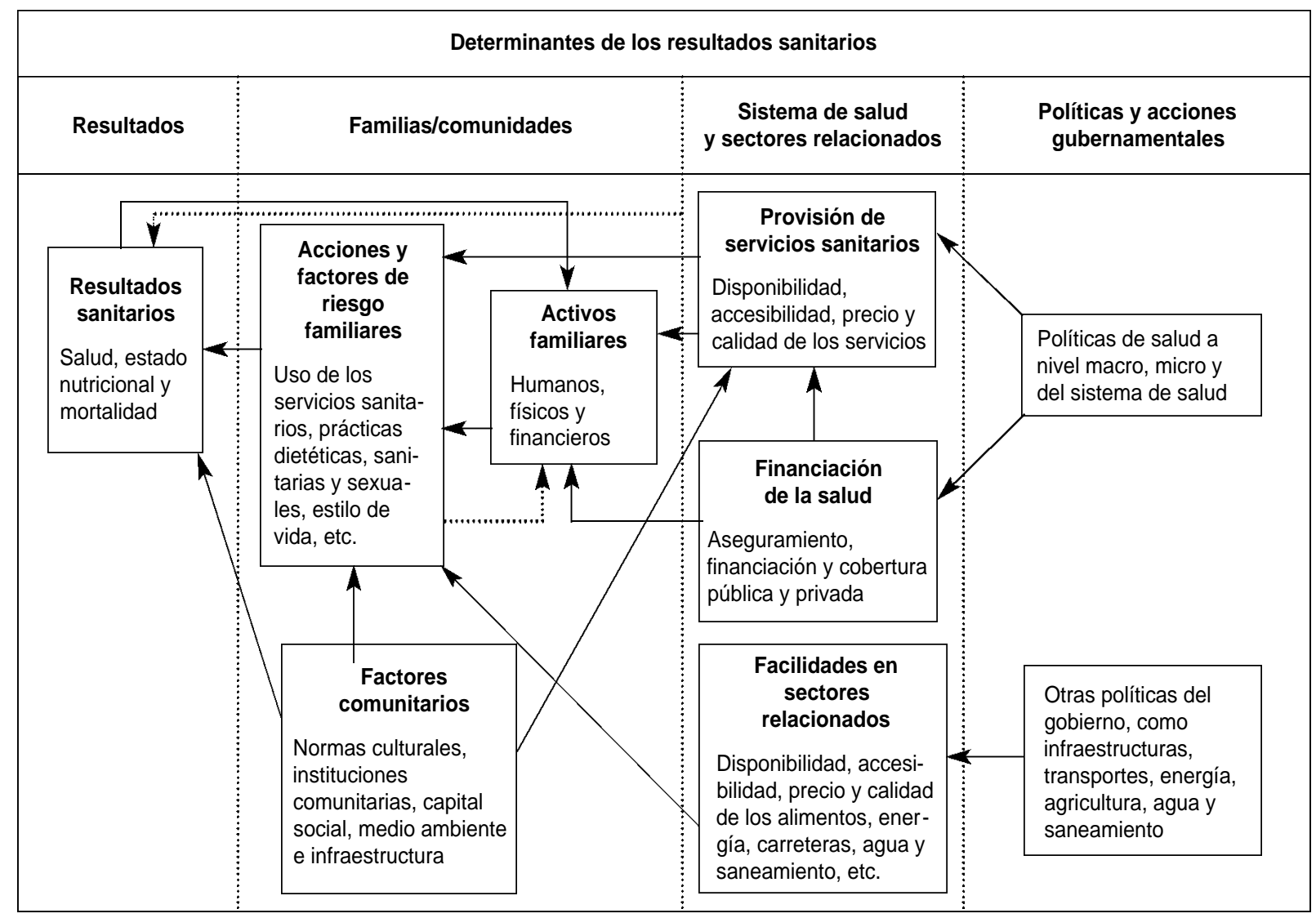

bres y los no pobres con respecto a la salud de los niños parecen ser mayores que en otras partes del mundo en desarrollo, cualquiera que sea el indicador sanitario utilizado. En cambio, las desigualdades en la mortalidad postinfantil (1 a 4 años) y la desnutrición son menos pronunciadas en el África Subsahariana que en el Norte de África, Asia y el Cercano Oriente, pero con la diarrea y las infecciones respiratorias agudas ocurre lo contrario (47).

Cuarto, las desigualdades socieconómicas en el campo de la salud parecen estar aumentando, en vez de disminuir, y esto es cierto tanto para el mundo en desarrollo (48-52) como para el mundo industrializado $(28,53-56)$.

\section{CAUSAS DE LAS DESIGUALDADES SANITARIAS: DETERMINANTES INMEDIATOS}

La figura 2 esboza una forma de conceptualizar las diferentes vías mediante las cuales se determinan los resultados de salud (7) y proporciona un marco para entender las desigualdades sanitarias entre los pobres y los pudientes.

\section{¿Cómo varían los determinantes inmediatos entre los grupos socioeconómicos?}

Los diferentes factores domésticos y comunitarios con influencia directa sobre los resultados sanitarios son llamados determinantes inmediatos de la salud en algunos círculos, (57) y en otros, entradas sanitarias en la producción de salud ${ }^{4}$ (58). Se sabe bastante sobre cuáles son y su etiología $(7,59)$. Varían mucho de un hogar a otro y tienden a ser peores en los hogares pobres que en los pudientes. Esto explica parcialmente por qué existen desigualdades socioeconómicas con respecto a la salud y por qué son desfavorables a los pobres. Sin embargo, las desigualdades en los determinantes inmediatos de la salud varían según los determinantes $\mathrm{y}$, al igual que las mismas desigualdades sanitarias, también según los países.

Esto es especialmente llamativo en el caso de la utilización de los servicios de salud. En los países de la OCDE los pobres tienden a utilizar los servicios de salud más que quienes tienen más dinero, $\mathrm{y}$

\footnotetext{
4 En el original, health inputs into the production of health.
} 
esto plantea la cuestión de si, en vista de sus mayores necesidades médicas, es suficiente la mayor utilización (60-65). En el mundo en desarrollo el cuadro es muy diferente. Típicamente los niños pobres de los países pobres tienen una probabilidad de estar vacunados muy inferior a la de los niños pudientes (39). Esto es así incluso en los países con programas nacionales de vacunación que proporcionan los servicios de forma gratuita en el punto de uso (66). La utilización de la rehidratación oral es menor entre los niños pobres que entre los de más dinero, aunque la incidencia de diarrea sea mayor en los primeros (39). En aquellos países en los que el uso de la rehidratación oral es mayor entre los pobres, esta desigualdad es mucho menor que la desigualdad con respecto a la incidencia de diarrea.

La incapacidad de los servicios de salud de los países en desarrollo para llegar a los pobres, a pesar de su mayor carga de enfermedad, no es únicamente una cuestión de que quienes tienen más dinero usen sus mayores ingresos para comprar atención en el sector privado. Los pobres también reciben menos de los subsidios gubernamentales al sector de la salud (67-71). El sesgo a favor de los ricos es especialmente marcado en el sector hospitalario, que se beneficia de la mayor parte del gasto del gobierno. Sin embargo, algunos países en desarrollo, como Costa Rica y Malasia, parecen ser capaces de conseguir distribuciones del gasto público en asistencia sanitaria más favorables a los pobres (47). En la India, el Estado de Kerala consigue asegurar una distribución de los subsidios sanitarios más o menos homogénea entre los grupos de ingresos (72).

Parece haber menos datos cuantitativos sobre el grado de desigualdad con respecto a otros determinantes inmediatos de la salud. La prevalencia de la lactancia materna suele ser mayor entre los grupos socioeconómicos más bajos (48), pero no parece ocurrir lo mismo con otros determinantes inmediatos de la salud infantil. El consumo de alcohol es mayor entre los grupos socioeconómicos más bajos de varios países de Europa oriental, Finlandia y Francia (73). El consumo de tabaco y las malas dietas tienden a concentrarse en los grupos socioeconómicos más bajos en los Estados Unidos de América y el norte de Europa, pero no en el sur de Europa y Francia (73). Entre los negros sudafricanos hay una asociación positiva entre el consumo de tabaco y el estatus socioeconómico, mientras que entre los blancos ocurre lo contrario (74).

\section{Contribución de los determinantes inmediatos a las desigualdades}

El simple hecho de saber que la distribución de este o aquel determinante inmediato desfavo- rece a los pobres no nos dice cuán importante es esa desigualdad para explicar las desigualdades sanitarias. La contribución de un determinante inmediato concreto a la desigualdad sanitaria depende en parte de su distribución entre los grupos socioeconómicos y en parte de su repercusión en la salud (52). El estudio Whitehall de los funcionarios públicos británicos evaluó la contribución relativa de varios determinantes inmediatos de la salud a las desigualdades sanitarias. North et al. (75) intentaron explicar la fuerte relación inversa entre la categoría del empleo y el absentismo laboral por motivos de salud. Se identificaron varios factores de riesgo, como los comportamientos relacionados con la salud, las características del trabajo, los bajos niveles de satisfacción con el trabajo y las circunstancias sociales adversas extralaborales. Los métodos de estandarización revelaron que las desigualdades con respecto a estos factores de riesgo solo explicaban aproximadamente un tercio de las diferencias de absentismo entre categorías laborales. Marmot et al. (76) realizaron un ejercicio similar con respecto a la cardiopatía coronaria.

\section{CAUSAS DE DESIGUALDADES SANITARIAS: DETERMINANTES SUBYACENTES}

¿Por qué hay desigualdades en los determinantes inmediatos de la salud? La figura 2 muestra la influencia de los recursos familiares, los factores comunitarios y los determinantes relacionados con el sistema de salud. Los pobres tienden a estar en desventaja con respecto a cada uno de estos determinantes subyacentes de la salud $(57,77,78)$.

\section{¿Cómo varían los determinantes subyacentes entre los grupos socioeconómicos?}

Los ingresos y los activos, cuyas desigualdades varían mucho según los países (79), son componentes clave de los recursos familiares. En los países en desarrollo, manteniendo constantes todas las demás condiciones, los mayores ingresos se asocian al uso más frecuente e intensivo de los servicios de salud, tanto en el sector privado como en el sector público (67); al uso de los servicios de profesionales sanitarios modernos, y no de curanderos tradicionales (67), y al número de hijos de cada mujer y a la edad a la que tiene su primer hijo. A medida que aumentan los ingresos también mejoran la mayoría de las prácticas dietéticas y de alimentación de los niños, así como las prácticas higiénicas (por ejemplo, el lavado de las manos y la eliminación de las heces). Los activos humanos de conocimiento, alfa- 
betización y educación, cuyos niveles tienden a ser más bajos entre los pobres $(39,80)$, también influyen sobre las decisiones familiares con respecto a los determinantes inmediatos de la salud. La educación, y en especial la educación de las mujeres, está fuertemente asociada a muchos comportamientos y elecciones que propician la buena salud, incluso después de controlar el efecto de los ingresos (77). Pero no son solo los niveles de estas variables lo que importa, sino también su distribución en cada hogar, especialmente entre los hombres y las mujeres. El escaso control de las mujeres sobre los recursos familiares, que parece ser especialmente frecuente en los hogares pobres, a menudo afecta negativamente los resultados sanitarios de sus familias y de ellas mismas (7).

\section{Factores comunitarios}

Por lo que respecta a los factores comunitarios, es importante considerar las influencias ambientales y geográficas. Es más difícil llegar a un centro de salud si los caminos se hacen intransitables durante la época de las lluvias. El ambiente también es importante. Es relativamente difícil mantener buenas prácticas sanitarias cuando la comunidad local dispone de malas condiciones de saneamiento y suministro de agua. Las comunidades suelen compartir valores y normas similares, que, a través de la presión de los demás, desempeñan a menudo un importante papel en la conformación de las conductas sanitarias (81). Los pobres tienen mayores probabilidades de estar en desventaja a nivel tanto de comunidad como de domicilio. Por ejemplo, tienen mayores probabilidades de vivir en zonas aisladas. Además, en las comunidades pobres, las presiones sociales entre los adolescentes tienden a ser más fuertes y las actitudes hacia las mujeres menos favorables a los buenos resultados sanitarios (81).

Hay bastantes datos sobre las repercusiones de los determinantes relacionados con el sistema de salud en los resultados sanitarios y la utilización de los servicios de salud. La disponibilidad, posiblemente definida como el personal existente en los servicios de salud locales, aparece a menudo como un importante determinante de la utilización de los servicios y de los resultados sanitarios (82-84). La accesibilidad, esto es, la facilidad con que las personas pueden acceder a los servicios, también es importante. En este aspecto es importante el tiempo de viaje, que depende de la distancia que hay que viajar, del sistema de transporte, de la infraestructura viaria y de factores geográficos. La distancia es la variable que más aparece en los estudios empíri- cos de utilización y suele tener una gran repercusión en ella $(82,85-88)$. El mayor precio monetario tiende a reducir la utilización, o al menos a aplazarla, sobre todo entre los pobres, a no ser que se acompañe de un mejoramiento de la calidad del servicio $(89,90)$. El seguro tiende a aumentar el uso de los servicios de salud $(91,92)$. La calidad o, más exactamente, la calidad percibida, también incrementa la demanda de servicios de salud $(82,88,89$, 93). Los pobres están en desventaja en la mayoría de estas áreas. Tienden a tener que viajar más lejos (93) y durante más tiempo (67) con el fin de llegar a los servicios de salud. La calidad de la atención, interpretada en un sentido amplio, que además de la calidad técnica incluye el servicio y las comodidades, también tiende a ser comparativamente menor en los servicios a disposición de los pobres (87). Los pobres, que son los usuarios de los servicios de salud más sensibles al precio, se enfrentan frecuentemente a un mayor precio en el punto de uso debido a que tienen menos probabilidades de disponer de seguros, sean privados (91) o públicos (94). A veces esta tendencia es anulada por los sistemas de exención de honorarios, aunque en la práctica estos suelen tener el efecto de eximir a los casi pobres, más que a los pobres $(90,94,95)$.

\section{Contribución de los determinantes subyacentes a las desigualdades}

Como ocurre con los determinantes inmediatos de la salud, el simple hecho de saber que la distribución de este o aquel determinante subyacente desfavorece a los pobres no indica cuán importante es esa desigualdad para explicar las desigualdades sanitarias. Una forma de encarar esta cuestión es el método usado en el estudio Whitehall (75). Otra consiste en usar un análisis de descomposición que, a través de un modelo de regresión de los determinantes de la salud, relacione las desigualdades en los varios determinantes de la salud con una medida de las desigualdades sanitarias (52).

En Cebú, Filipinas, se usó este método para desvelar las causas subyacentes de las desigualdades con respecto a la supervivencia infantil (96). Se identificaron varios determinantes importantes de la supervivencia infantil, como la educación de la madre, los ingresos familiares, la cobertura por seguro de salud, la disponibilidad de agua potable, las condiciones de saneamiento, el tiempo o la distancia de viaje a varias instalaciones de los servicios de salud, el personal disponible en los servicios locales de atención primaria y la disponibilidad local de fármacos clave. Con respecto a su contribución a las desigualdades de supervivencia entre los niños 
pobres y no pobres, el factor más importante de todos estos fue el ingreso. Las desigualdades con respecto a la educación de la madre también fueron importantes. Las desigualdades con respecto a la disponibilidad de servicios de salud fueron relativamente pequeñas, de forma que, aunque tuvieron una importante influencia sobre las perspectivas de supervivencia media de los niños, no ayudaron a explicar las diferencias de supervivencia entre los niños pobres y los no pobres. Se ha usado el mismo método para investigar las causas del aumento de las desigualdades con respecto a la malnutrición en Vietnam entre 1993 y 1998 (52) y las causas de las desigualdades con respecto a la autoevaluación de la salud en personas de 33 años del Reino Unido (97). Al igual que en el estudio de Cebú, en estos dos estudios las desigualdades con respecto a variables relacionadas con el individuo (por ejemplo, la educación) y con el hogar (por ejemplo, los ingresos, la vivienda, la disponibilidad de agua potable segura y el saneamiento) explicaron, en su conjunto, gran parte de las desigualdades sanitarias.

\section{POBREZA Y PAGO POR LA ATENCIÓN SANITARIA}

Además de su preocupación por mejorar la salud de los pobres, la colectividad que trabaja en el campo del desarrollo internacional también está preocupada por la repercusión de los costos de la atención sanitaria y de los ingresos perdidos en la capacidad de las familias para comprar otras cosas que no sean atención sanitaria. En otras palabras, además del deseo de asegurar que mejore la salud, especialmente entre los pobres, también hay un deseo de asegurar que la consecución de este objetivo no se haga a costa de una reducción excesiva del nivel de vida de las familias implicadas.

Hay varias formas posibles de entender estas cuestiones (21). Una es que la distribución de los costos de la atención sanitaria no se debe hacer de forma que aumente la desigualdad de los ingresos. Los pagos regresivos, esto es, los que absorben una parte mayor de los ingresos de las familias pobres que de las familias ricas, violan este requisito. Los pagos de bolsillo son regresivos en la mayoría de los países de la OCDE $(98,99)$ y en algunos países en desarrollo, como las regiones rurales de Bangladesh (100), Burkina Faso (101), China (102), Paraguay (101), Sierra Leona (103) y Tailandia (104). Sin embargo, en varios países en desarrollo son proporcionales a los ingresos, como ocurre en Vietnam $(21,105)$, o progresivos, como en Guatemala (101), India (72), México (106), Nepal (107) y Sudáfrica (101). En el primer grupo de países los pobres pare- cen utilizar los servicios, pero pagan por ellos una cuota mayor de sus ingresos, mientras que en el último grupo son principalmente los pudientes quienes usan y pagan los servicios de salud. La preocupación por la regresividad de los pagos de bolsillo no toma en consideración la posibilidad de que esta pueda ser compensada, al menos en parte, por la progresividad de los prepagos, esto es, los impuestos, las contribuciones a la seguridad social y las primas de los seguros privados. En muchos países de la OCDE, la progresividad de estos pagos indirectos es, de hecho, más que suficiente para compensar la regresividad de los pagos directos (98).

Una segunda interpretación de la cuestión de los pagos por la atención sanitaria es que las familias no deberían gastar en ella más que un porcentaje determinado de sus ingresos y que los pagos superiores a este umbral se clasificaran como catastróficos (21). En varios países, más del 1\% de las familias habían gastado recientemente en atención sanitaria la mitad o más de sus desembolsos no alimentarios (24). Otro estudio reciente exploró las tendencias de los gastos sanitarios catastróficos en Vietnam, comprobándose que, independientemente del umbral fijado y de si los gastos se calcularon como cuota de los gastos totales o de los gastos no alimentarios, la proporción de la población que hizo pagos catastróficos disminuyó entre 1993 y 1998 (21).

Una tercera interpretación es que los costos de la atención sanitaria no deberían llevar a las familias a la pobreza ni aumentarla. El impacto sobre la pobreza puede medirse por el cambio en el recuento de pobres (esto es, la proporción de la población en estado de pobreza) o por el cambio en la brecha de pobreza (esto es, la diferencia media con respecto a la línea de pobreza) inducido por los pagos de la atención sanitaria (21). Con la brecha de pobreza es posible distinguir entre las personas ya pobres que se hacen todavía más pobres y los que se hacen pobres no siéndolo antes. Los cálculos realizados con estos métodos han indicado que los gastos de bolsillo en atención hospitalaria podrían haber aumentado el recuento de pobres en un $2 \%$ en la India (72) y que, para una línea de pobreza basada en los alimentos, los gastos globales en atención sanitaria en Vietnam podrían haber aumentado el recuento de pobres en cerca de un $4,4 \%$ en 1993 y de un 3,4\% en 1998 (21). En Vietnam, el impacto sobre la brecha de pobreza fue bastante menor que en el recuento de pobres $(1,4 \%$ en 1993 y $0,8 \%$ en 1998) y tres cuartas partes de la misma fueron atribuibles a los ya pobres que se hicieron más pobres. En Vietnam, la mayor parte del impacto de los gastos de bolsillo sobre la pobreza fueron atribuibles a los gastos no hospitalarios. 


\section{DESIGUALDADES EN EL SECTOR DE LA SALUD Y POLÍTICAS PÚBLICAS}

\section{Estudios generales de los efectos de las políticas}

En un estudio comparativo de nueve países de la OCDE, la desigualdad con respecto a la autoevaluación de la salud no se asoció significativamente a los gastos totales en atención sanitaria per cápita, al porcentaje de la contribución pública ni al producto interior bruto per cápita, pero sí, de forma significativa y positiva, a la desigualdad de ingresos (36). Sin embargo, en otra investigación en la que se utilizaron datos agregados de países en desarrollo y un método descompositivo, se observó que el gasto público en salud tenía mayor impacto sobre la mortalidad infantil entre los pobres que entre los no pobres, sirviendo, por lo tanto, para reducir la desigualdad con respecto a la salud (108). En otro estudio comparativo se verificó que las diferencias entre los países de la OCDE con respecto a la magnitud de la desigualdad e inequidad en la utilización de la atención sanitaria refleja, en parte, diferencias entre los pobres y los pudientes con respecto a los honorarios pagados por el usuario, pero no en el alcance de la cobertura del seguro (63). Este estudio también obtuvo pruebas de que la distribución de la utilización entre grupos de ingreso reflejaba algunas características del sistema de prestación, como la forma de pago de los proveedores, pero no otras, como la existencia de un sistema de derivación por un médico general. En otro estudio de países de la OCDE se informó que la progresividad de los pagos combinados, directos e indirectos, por atención sanitaria reflejan fielmente la mezcla financiera del sistema. En los sistemas financiados por impuestos, en términos generales los pagos tendieron a ser proporcionales a los ingresos; en los sistemas de seguridad social, en el peor de los casos tendieron a ser ligeramente regresivos, pero a veces eran proporcionales o incluso ligeramente progresivos, y en los sistemas con financiación predominantemente privada tendieron a ser regresivos (98).

\section{Efectos de programas específicos}

Yip y Berman (109) examinaron las desigualdades en la cobertura del seguro entre niños pobres y pudientes con el Programa de Seguro de Salud Escolar (SHIP: School Health Insurance Programme) de Egipto. Además exploraron las diferencias exógenas con respecto a la cobertura del seguro de salud, con el fin de evaluar el impacto del programa en la distribución tanto de la utilización de los servicios de salud como de los pagos de bolsillo. Aun- que el SHIP estaba destinado a cubrir a todos los niños que estuvieran estudiando, esto es, los de 6 a 18 años, en el momento en el que se realizó la encuesta algunos niños escolarizados todavía no estaban cubiertos. Estos niños constituían un grupo de control, pero los autores usaron el análisis de regresión para controlar otras diferencias entre los niños cubiertos y los no cubiertos. La cobertura del SHIP aumentó con los ingresos, sobre todo debido a que los niños más pobres tenían menos probabilidades de estar escolarizados, pero también porque los niños escolarizados que todavía no tenían cobertura tenían mayores probabilidades de ser pobres. La cobertura por el SHIP aumentó la probabilidad de que se consultara a un profesional sanitario formal en todos los grupos de ingresos, pero el impacto fue especialmente grande entre los niños del quintil más pobre. La cobertura por el programa originó menores pagos de bolsillo en todos los grupos de ingreso, pero el impacto fue mucho menor en los quintiles más pobre y más rico que en el medio de la distribución de ingresos.

Victora et al. (48) han presentado datos sobre el programa de salud maternoinfantil de Ceará, Brasil, destinado específicamente a reducir las desigualdades sanitarias. Tras la introducción del programa se observaron considerables mejorías de los niveles medios de utilización de los servicios y de los resultados. Sin embargo, aunque hubo una disminución de las desigualdades con respecto a la cobertura de vacunación, al peso y al uso de la rehidratación oral, también hubo un aumento de la desigualdad entre los niños pobres y los más acomodados con respecto a las tres variables estudiadas. Los autores también examinaron el impacto conjunto de varios programas puestos en funcionamiento en la ciudad de Pelotas, Brasil, entre ellos un gran aumento del número de servicios de salud gubernamentales de primera línea, la creación de tres unidades de cuidados neonatales y un aumento general del gasto del gobierno en servicios preventivos y curativos. A lo largo del período estudiado, aumentaron las proporciones de embarazadas que recibieron atención prenatal y de niños que recibieron tres dosis de la vacuna contra el tétanos, la difteria y la tos ferina (DPT) en el primer año de vida, mientras que las desigualdades en la utilización de estos servicios disminuyeron. Además, las disminuciones de las tasas medias de mortalidad infantil y de malnutrición se acompañaron de reducciones de las desigualdades con respecto a estas variables.

En este campo son relativamente raras las comparaciones antes-después con grupos de control, una forma más eficaz de establecer el impacto de los programas que los simples estudios antesdespués. Un estudio de este tipo fue el de Bhuiya et 
al. (110), que analizó el impacto de un programa de salud maternoinfantil, llevado a cabo por el Centro Internacional de Enfermedades Diarreicas de Bangladesh, sobre las diferencias de mortalidad de los niños de 1 a 5 años. En algunas áreas no cubiertas por el programa, esto es, en el grupo de control, solo se proporcionaron servicios de salud gubernamentales, y en otras había una organización no gubernamental (conocida como BRAC: Bangladesh Rural Advancement Committee) que tenía en funcionamiento un programa de desarrollo socioeconómico que se sumaba a los servicios de salud prestados por el gobierno. El programa del BRAC estaba destinado a la reducción de la pobreza y al desarrollo de la mujer, y la mayoría de sus actividades, entre las que se incluía la atención sanitaria básica, iban dirigidas a las mujeres pobres. Durante el período de estudio, ambas áreas mostraron una reducción de las tasas de mortalidad postinfantil ligeramente superior al $40 \%$, reducción que fue un poco mayor en el área de control. No obstante, la distribución de las reducciones entre los grupos de ingresos fue muy diferente. En el área de control la mayor reducción porcentual correspondió al grupo más rico, mientras que en el área donde estaba funcionando el programa de salud maternoinfantil las mayores reducciones ocurrieron en el grupo más pobre.

Diop et al. realizaron una comparación "antesdespués" de dos distritos de Níger en los que se introdujeron reformas de la financiación a principios de la década de los 90 (111). Antes del comienzo del estudio se mejoró la disponibilidad de medicamentos, se preparó al personal sanitario y se fortaleció la capacidad de gestión y supervisión. En el distrito de Say se introdujeron honorarios a pagar por los usuarios, con exenciones para algunas categorías, y los ingresos procedentes de esos honorarios se usaron para financiar productos farmacéuticos y crear un fondo de solidaridad. En el distrito de Boboye se establecieron menores honorarios, pero se creó un impuesto local destinado al fondo sanitario distrital. En el distrito de Illéla, que sirvió como control, no se efectuaron cambios. La utilización de los servicios de salud públicos disminuyó a lo largo del período de estudio en el cuartil más pobre de la población, tanto en Illéla como en Say. La disminución fue mayor en Say, donde se cobraron honorarios, y en este distrito la disminución de la utilización fue proporcionalmente mayor en el cuartil más pobre de la población que en la totalidad de la misma. No obstante, los cambios en la utilización de servicios por el cuartil más pobre no fueron estadísticamente significativos en ninguno de los dos distritos. Por el contrario, con el sistema de "honorario e impuesto" de Boboye, la utilización se duplicó en el cuartil más pobre de la población. Este cambio, estadísticamente significativo, fue mucho mayor que el de la totalidad de la población del distrito.

\section{CONCLUSIONES}

Primero, sabemos bastante sobre el alcance de las desigualdades sanitarias entre los pobres y los no pobres en los países en desarrollo, y tenemos un conocimiento razonable de las desigualdades con respecto a los factores determinantes de la salud. Lo más llamativo en este aspecto es la incapacidad de la atención sanitaria con financiación pública para llegar a los pobres en casi todos los países en desarrollo, problema que merece una seria atención por parte de los gobiernos y de los organismos de ayuda.

Segundo, se sabe muy poco sobre la importancia relativa de las desigualdades con respecto a los factores determinantes de la salud y a la utilización de los servicios de salud. Lo que sabemos indica que las desigualdades sanitarias, y muy probablemente también en la utilización de los servicios, reflejan en gran medida las desigualdades con respecto a variables tanto individuales como familiares, tales como la educación, los ingresos, la localización y las características de los hogares. Esto indica que las políticas destinadas a combatir las desigualdades en el sector de la salud deberían dirigirse a reducir las desigualdades tanto en el lado de la oferta (por ejemplo, la calidad y la disponibilidad de servicios de salud), como en el lado de la demanda (ingresos, conocimientos, y especialmente conocimientos relacionados específicamente con la salud, accesibilidad de los servicios de salud, disponibilidad de agua potable segura, saneamiento, etc.). Los ministerios de salud deberían colaborar más con otros ministerios, pero también deberían adoptar una visión más amplia, por ejemplo, explorando métodos alternativos de prestación para llegar a los pobres y encontrando mejores métodos de aumentar los conocimientos de los pobres acerca de los comportamientos saludables.

Tercero, se sabe muy poco sobre el impacto de los programas y las políticas en las desigualdades en el sector sanitario. Indudablemente, hay una gran laguna en nuestro conocimiento sobre cuál es la mejor forma de llegar a los pobres en el sector de la salud. Para cubrir esta laguna son necesarios más trabajos en la línea de los estudios antes mencionados, relacionados con las desigualdades en el sector de la salud y las políticas públicas.

Agradecimientos. A George Alleyne por haberme invitado a escribir este artículo para la Co- 
misión sobre Macroeconomía y Salud, y a mis coautores del capítulo sobre salud, nutrición y población del libro del Banco Mundial "Estrategia de reducción de la pobreza", en el cual me he basado.
También agradezco a los dos revisores anónimos y a los editores del Bulletin of the World Health Organization por sus útiles comentarios a una versión anterior.

\section{REFERENCIAS}

1. World Bank. Confronting AIDS: public priorities in a global epidemic. Oxford: Oxford University Press; 1999.

2. Bloom D, Sachs J. Geography, demography and economic growth in Africa. Brookings Papers on Economic Activity 1998;2:207-295.

3. Eastwood R, Lipton M. The impact of changes in human fertility on poverty. J Dev Stud 1999;36:1-30.

4. Narayan D, Patel R, Schafft K, Rademacher A, Koch-Schulte S. Voices of the poor: can anyone hear us? New York: Oxford University Press; 2000.

5. Pritchett L, Summers LH. Wealthier is healthier. J Hum Resour 1996;31:841868.

6. World Bank. World development report 2000/2001: attacking poverty. Oxford and New York: Oxford University Press; 2000.

7. Claeson M, Griffin CG, Johnston TA, McLachlan M, Soucat ALB, Wagstaff A, et al. Health, nutrition and population. In: Poverty reduction strategy sourcebook. Washington, D.C.: World Bank; 2001.

8. World Bank. Health, nutrition and population sector strategy. Washington, D.C.: World Bank; 1997.

9. World Health Organization. The world health report 1999. Making a difference. Geneva: WHO; 1999.

10. Department for International Development. Better health for poor people. London: Department for International Development; 1999.

11. World Bank. What is the World Bank? Washington, D.C.: [Sitio en Internet] World Bank. Disponible en: www. worldbank.orgabout/whatis/. (Acceso el 3 enero 2002).

12. Department for International Development. Eliminating world poverty: a challenge for the 21st century. London: The Stationery Office; 1997. White Paper on international development.

13. Sen A. Development as freedom. New York: Knopf; 1999

14. Department of Health. Tackling health inequalities: consultation on a plan for delivery. London: Department of Health; 2001.

15. Le Grand J. Equity, health and health care. Soc Justice Res 1987; 1:257-274.

16. Alleyne GAO, Casas J, Castillo-Salgado C. Equality, equity: why bother? Bull World Health Organ 2000;78:76-77.

17. Wagstaff A. Economics, health and development: some ethical dilemmas fa- cing the World Bank and the international community. J Med Ethics 2001; 27:262-267.

18. Evans T, Whitehead M, Diderichsen F, Bhuiya A, Wirth M. Introduction. En: Evans T, Whitehead M, Diderichsen F, Bhuiya A, Wirth M, eds. Challenging inequities in health: from ethics to action. Oxford: Oxford University Press; 2001.

19. Whitehead M. The concepts and principles of equity and health. Int J Health Serv 1992;22:429-445.

20. Braveman P, Starfield B, Geiger H. World health report 2000: how it removes equity from the agenda for public health monitoring and policy. BMJ 2001;323:678-680.

21. Wagstaff A, van Doorslaer E. Paying for health care: quantifying fairness, catastrophe and impoverishment, with applications to Viet Nam 1993-98. Washington, D.C.: World Bank; 2001. Policy Research Working Paper No. 2715.

22. Organisation for Economic Co-operation and Development. The reform of health care: a comparative analysis of seven OECD countries. Paris: OECD; 1992.

23. Murray C, Knaul F, Musgrove P, Xu K, Kawabata K. Defining and measuring fairness in financial contribution to the health system. Geneva: World Health Organization; 2000. GPE Discussion Paper No. 24.

24. World Health Organization. The world health report 2000. Health systems: improving performance. Geneva: WHO; 2000.

25. Smith J. Healthy bodies and thick wallets: the dual relation between health and socioeconomic status. J Econ Perspect 1999;13:145-166.

26. Wagstaff A, Paci P, van Doorslaer E. On the measurement of inequalities in health. Soc Sci Med 1991;33:545-557.

27. Kakwani N, Wagstaff A, van Doorlsaer E. Socioeconomic inequalities in health: measurement, computation and statistical inference. J Econom 1997;77:87-104.

28. Mackenbach JP, Kunst AE. Measuring the magnitude of socio-economic inequalities in health: an overview of available measures illustrated with two examples from Europe. Soc Sci Med 1997;44:757-771.

29. Drever F, Whitehead M, eds. Health inequalities: decennial supplement. London: The Stationery Office; 1997. Series DS No. 15.
30. Fox A. Health inequalities in Europe. Aldershot: Gower; 1989.

31. Kunst AE, Geurts JJ, van den Berg J. International variation in socioeconomic inequalities in self reported health. J Epidemiol Community Health 1995;49: 117-123.

32. Mackenbach JP, Kunst AE, Cavelaars AEJM, Groenhof F, Geurts JJM, EU Working Group on Socioeconomic Inequalities in Health. Socioeconomic inequalities in morbidity and mortality in western Europe. Lancet 1997;349:16551659.

33. Marmot M, Smith GD, Stansfeld $S$, Patel C, North F, Head J, et al. Health inequalities among British civil servants: the Whitehall II study. Lancet 1991;337:1387-1393.

34. Townsend P, Davidson N. Inequalities in health: the Black report. Harmondsworth: Penguin; 1982.

35. Vagero D, Erikson R. Socioeconomic inequalities in morbidity and mortality in western Europe. Lancet 1997;350: 516-517.

36. Van Doorslaer E, Wagstaff A, Bleichrodt $\mathrm{H}$, Caonge S, Gerdtham U-G, Gerfin $\mathrm{M}$, et al. Income-related inequalities in health: some international comparisons. J Health Econ 1997; 16:93-112.

37. Whitehead M. William Farr's legacy to the study of health inequalities. Bull World Health Organ 2000;78:86-87.

38. Bonilla-Chacin M, Hammer J. Life and death among the poorest. Washington, D.C.: World Bank; 1999. (Unpublished document).

39. Gwatkin D, Rutstein S, Johnson K, Pande R, Wagstaff A. Socioeconomic differences in health, nutrition and population. Washington, D.C.: World Bank; 2000. Health, Nutrition and Population Discussion Paper.

40. Wagstaff A, Watanabe N. Socioeconomic inequalities in child malnutrition in the developing world. Washington, D.C.: World Bank; 2000. (Policy Research Working Paper No. 2434).

41. Wagstaff A. Socioeconomic inequalities in child mortality: comparisons across nine developing countries. Bull World Health Organ 2000;78:19-29.

42. Filmer D. Malaria among the poor and less poor in sub-Saharan Africa. Washington, D.C.: World Bank; 2001. Unpublished document.

43. Baker J, van der Gaag J. Equity in health care and health care financing: Evidence from five developing countries. 
En: Van Doorslaer E, Wagstaff E, Rutten $\mathrm{F}$, eds. Equity in the finance and delivery of health care: an international perspective. Oxford: Oxford University Press; 1993.

44. Van Doorslaer E, Wagstaff A, Rutten F, eds. Equity in the finance and delivery of health care: an international perspective. Oxford: Oxford University Press; 1993.

45. Wagstaff A. Poverty and health. Boston (MA): WHO Commission on Macroeconomics and Health; 2001. Working Group No.1, Working Paper No. 5.

46. Blaxter M. A comparison of measures of inequality in morbidity. En: Fox J, ed. Health inequalities in European countries. Aldershot: Gower; 1989. Pp. 199-230.

47. Wagstaff A. Inequalities in health in developing countries: swimming against the tide? Washington, D.C.: World Bank; 2001. Policy Research Working Paper.

48. Victora C, Barros F, Vaughan J, Silva A, Tomasi E. Explaining trends in inequities: evidence from Brazilian child health studies. Lancet 2000;356:10931098.

49. Wagstaff A, Nguyen N. Poverty and survival prospects of Vietnamese children under Doi Moi. Washington, D.C.: World Bank; 2001. Policy Research Working Paper.

50. Stecklov G, Bommier A, Boerma T. Trends in equity in child survival in developing countries: an illustrative analysis using Ugandan data. Chapel Hill (NC): Carolina Population Center, University of North Carolina at Chapel Hill; 1999. (Unpublished document).

51. Vega J, Hollstein R, Delgado I, Perez K, Carrasco S, Marshall G, et al. Chile: socioeconomic differentials and mortality in a middle-income nation. En: Evans T, Whitehead M, Diderichsen F, Bhuiya A, Wirth M, eds. Challenging inequalities in health: from ethics to action. Oxford: Oxford University Press; 2001.

52. Wagstaff A, van Doorslaer E, Watanabe N. On decomposing the causes of health sector inequalities, with an application to malnutrition inequalities in Viet Nam. Washington, D.C.: World Bank; 2001. Policy Research Working Paper No. 2714.

53. Graham $H$. The challenge of health inequalities. En: Graham H, ed. Understanding health inequalities. Buckingham and Philadelphia (PA): Open University Press; 2000.

54. Propper C, Upward R. Need, equity and the NHS: the distribution of health care expenditure 1974-87. Fiscal Studies 1992;13:1-21.

55. Schalick LM, Hadden WC, Pamuk E, Navarro V, Pappas G. The widening gap in death rates among income groups in the United States from 1967 to 1986. Int J Health Serv 2000;30:13-26.

56. Pappas G, Queen S, Hadden W, Fisher $\mathrm{G}$. The increasing disparity in mortality between socioeconomic groups in the United States, 1960 and 1986. N Engl J Med 1993;329:103-109.

57. Mosley W, Chen L. An analytical framework for the study of child survival in developing countries. Popul Dev Rev 1984; 10:25-45.

58. Grossman M. The demand for health: a theoretical and empirical investigation. New York: National Bureau of Economic Research; 1972.

59. World Health Organization. CHD 19961997 report. Geneva: WHO; 1998. Unpublished document $\mathrm{WHO} / \mathrm{CHD} / 98.5$.

60. Le Grand J. The distribution of public expenditure: the case of health care. Economica 1978;45:125-142.

61. Le Grand J. The strategy of equality: re distribution and the social services. London and Boston (MA): Allen and Unwin; 1982.

62. Van Doorslaer E, Wagstaff A, Calonge S, Christiansen T, Gerfin M, Gottschalk $\mathrm{P}$, et al. Equity in the delivery of health care: some international comparisons. J Health Econ 1992;11: 389-411.

63. Van Doorslaer E, Wagstaff A, van der Burg H, Christiansen T, De Graeve D, Gerdtham U-G, et al. Equity in the delivery of health care in Europe and the US. J Health Econ 2000;19:553-584.

64. Wagstaff A, Van Doorslaer E, Paci P. On the measurement of horizontal inequity in the delivery of health care. J Health Econ 1991;10:169-205.

65. Wagstaff A, van Doorslaer E. Measuring and testing for inequity in the delivery of health care. J Hum Resour 2000;35: 716-733.

66. Pande R, Yazbeck A. What's in a country average? Income, gender, and regional inequalities in immunization in India. Washington, D.C.: World Bank; 2001. (Unpublished document).

67. Castro-Leal F, Dayton J, Demery L, Mehra K. Public social spending in Africa: Do the poor benefit? World Bank Res Obs 1999;14:49-72.

68. Castro-Leal F, Dayton J, Demery L, Mehra K. Public spending on health care in Africa: do the poor benefit? Bull World Health Organ 2000;78:66-74.

69. Filmer D, Hammer J, Pritchett L. Health policy in poor countries: weak links in the chain. Washington, D.C.: World Bank; 1998. (Policy Research Working Paper No.1874).

70. Sahn D, Younger S. Expenditure incidence in Africa: microeconomic evidence. Fiscal Studies 2000;21:329-348.

71. Yaqub S. How equitable is public spending on health and education? Washington, D.C.: World Bank; 1999. Background Paper for World Development Report 2000/1.

72. World Bank. Raising the sights: better health systems for India's poor. Washington, D.C.: World Bank; 2001.

73. Kunst A. Cross-national comparisons of socioeconomic differences in mortality. The Hague: CIP-Gegevens Koninklijke Bibliotheek; 1997.
74. Marmot M, Mustard J. Coronary heart disease from a population perspective. En: Evans R, Barer M, Marmor T, eds. Why are some people healthy and others not? New York: Aldine de Gruyter; 1994.

75. North F, Syme SL, Feeney A, Head J, Shipley MJ, Marmot MG. Explaining socioeconomic differences in sickness absence: the Whitehall II study. BMJ 1993;306:361-366.

76. Marmot M, Rose G, Shipley M, Hamilton P. Employment grade and coronary heart disease in British civil servants. J Epidemiol Community Health 1978;32: 244-249.

77. Cebu Study Team. Underlying and proximate determinants of child health: The Cebu longitudinal health and nutrition study. Am J Epidemiol 1991;133:185-201.

78. Schultz T. Studying the impact of household economic and community variables on child mortality. Popul Dev Rev 1984; 10:215-235.

79. World Bank. World development indicators 2001. Washington, D.C.: World Bank; 2001.

80. Filmer D, Pritchett L. The effect of household wealth on educational attainment: evidence from 35 countries. Popul Dev Rev 1999;25:85-120.

81. Woolcock M, Narayan M. Social capital: implications for development theory, research and policy. World Bank Res Obs 2000;15:225-249.

82. Lavy V, Strauss J, Thomas D, de Vreyer P. Quality of care, survival and health outcomes in Ghana. J Health Econ 1996; 15:333-357.

83. Panis C, Lillard L. Health inputs and child mortality. J Health Econ 1994;13: 455-489.

84. Rosenzweig M, Wolpin K. Governmental interventions and household behavior in a developing country: anticipating the unanticipated consequences of social programs. J Dev Econ 1982;10: 209-225.

85. Benefo K, Schultz T. Fertility and child mortality in Côte d'Ivoire and Ghana. World Bank Econ Rev 1996;10:123-158.

86. Mwabu G, Ainsworth M, Nyamete A. Quality of medical care and choice of medical treatment in Kenya: an empirical analysis. J Hum Resour 1993;28: 838-862.

87. Thomas D, Lavy V, Strauss D. Public policy and anthropometric outcomes in the Côte d'Ivoire. J Public Econ 1996;61: 155-192.

88. Wong E, Popkin B, Guilkey D, Akin J. Accessibility, quality of care and prenatal care use in the Philippines. Soc Sci Med 1987;24:927-944.

89. Alderman H, Lavy V. Household responses to public health services: cost and quality tradeoffs. World Bank Res Obs 1996; 11:3-22.

90. Gilson L. The lessons of user fee experience in Africa. Health Policy Plan 1997;12:273-285 
91. Gertler P, Sturm R. Private health insurance and public expenditures in Jamaica. J Econom 1997;77:237-258.

92. Schwartz J, Akin J, Popkin B. Price and income elasticities of demand for modern health care: the case of infant delivery in the Philippines. World Bank Econ Rev 1988;2:49-76.

93. Akin J, Hutchinson P. Health care facility choice and the phenomenon of bypassing. Health Policy Plan 1999;14: 135-151.

94. World Bank, Swedish International Development Cooperation Agency, Australian Agency for International Development, Royal Netherlands Embassy, Ministry of Health of Viet Nam. Growing healthy: a review of Viet Nam's health sector. Hanoi: World Bank; 2001.

95. Leighton C, Diop F. Protection of the poor under cost recovery. Bethesda (MD): Abt Associates; 1999. (Unpublished document).

96. Wagstaff A. Unpacking the causes of inequalities in child survival: the case of Cebu, the Philippines. Washington, D.C.: World Bank; 2000. (Unpublished document).

97. Wagstaff A, Paci P, Joshi $H$. Inequalities in health: Who you are? Where you live? Or who your parents were? Evidence from a cohort of British 33-yearolds. Washington, D.C.: World Bank; 2001. Policy Research Working Paper No.2713.

98. Wagstaff A, van Doorslaer E, van der Berg $\mathrm{H}$, Calonge S, Christiansen T, Ci- toni G. Equity in the finance of health care: some further international comparisons. J Health Econ 1999;18:263290.

99. Van Doorslaer E, Wagstaff A, van der Berg H, Christiansen T, Citoni G, Di Biase $R$, et al. The redistributive effect of health care finance in twelve OECD countries. J Health Econ 1999;18:291314.

100. Sen B. Health and poverty in the context of country development strategy: a case study on Bangladesh. Geneva: World Health Organization; 1997. Macroeconomics and Health Development Series No. 26. Unpublished document WHO/ ICO/MESD.26.

101. Makinen M, Waters H, Rauch M, Almagambetova N, Bitran R, Gilson L, et al. Inequalities in health care use and expenditures: empirical data from eight developing countries and countries in transition. Bull World Health Organ 2000;78:55-65.

102. Gao J, Tang S, Tolhurst R, Rao K. Changing access to health services in urban China: implications for equity. Health Policy Plan 2001;16:302-312.

103. Fabricant SJ, Kamara CW, Mills A Why the poor pay more: household curative expenditures in rural Sierra Leone. Int J Health Plann Manage 1999;14:179-199.

104. Pannarunothai S, Mills A. The poor pay more: health-related inequality in Thailand. Soc Sci Med 1997;44:17811790 .
105. Wagstaff A. Measuring equity in health care financing: reflections on and alternatives to WHO's fairness of financing index. Washington, D.C.: World Bank; 2000. (Policy Research Working Paper No. 2550).

106. Parker S, Pier E. Mexico. En: Greene E, Zevallos J, Suarez R, eds. Health systems inequalities and poverty in Latin America and the Caribbean. Washington, D.C.: Pan American Health Organization/World Bank; 1999.

107. Hotchkiss DR, Rous JJ, Karmacharya K, Sangraula P. Household health expenditures in Nepal: implications for health care financing reform. Health Policy Plan 1998;13:371-383.

108. Bidani B, Ravallion M. Decomposing social indicators using distributional data. J Econom 1997;77:125-139.

109. Yip W, Berman P. Targeted health insurance in a low income country and its impact on access and equity in access: Egypt's school health insurance. Health Econ 2001;10:207-220.

110. Bhuiya A, Chowdhury M, Ahmed F, Adams A. Bangladesh: an intervention study of factors underlying increasing equity in child survival. En: Evans T, Whitehead M, Diderichsen F, Bhuiya A, Wirth $\mathrm{M}$, eds. Challenging inequities in health: from ethics to action. Oxford: Oxford University Press; 2001.

111. Diop F, Yazbeck A, Bitran R. The impact of alternative cost recovery schemes on access and equity in Niger. Health Policy Plan 1995;10:223-240.

\section{Equidad Listserv Electronic Discussion Group for Information on Equity, Health, and Human Development}

The Equidad Listserv is an electronic discussion group that disseminates information on equity, health, and human development via Internet e-mail messages. Information is posted in English, Spanish, and/or Portuguese. The information service is administered by the Division of Health and Human Development of the Pan American Health Organization.

List subscribers can post messages to the other subscribers and tell them about new documents, reports, projects, project results, upcoming conferences and mee tings, bibliographies, Internet resources, and other useful materials. Themes fre quently covered in the postings include research, public policies, health legislation, de mocracy, governance, health determinants, health economics, sociology, poverty, ethics, and gender equity.

If you want to subscribe to the list so you can receive the new messages posted to it and post ones of your own, send an e-mail message to: listserv@paho.org. In the subject line of the e-mail message, type the word "Subscribe." In the body of the message, type the words "SUBSCRIBEEQUIDAD" and also your full name. You will re ceive an e-mail message confirming your subscription request. Once you are subscribed, you can also view an archive of earlier messages posted to the list. 